\title{
A network of reception of over-horizon VHF signals associated with earthquakes and some preliminary results
}

\author{
M. Hayakawa ${ }^{1)}$, T. Gotoh ${ }^{1)}$, and M. Ikeda ${ }^{2)}$ \\ 1) The University of Electro-Communications, Department of Electronic Engineering, 1-5-1 \\ Chofugaoka, Chofu, Tokyo 182-8585, Japan \\ 2) Fujimi-cho 20-2, Hachioji, Tokyo 192-0044, Japan
}

\begin{abstract}
The reception of over-horizon VHF signal is recently found to be useful for the study of seismo- atmospheric perturbation. So, we have extended our present single path from Chofu to FM Sendai to a nation-wide network. This paper describes our newly established network for reception of over-horizon VHF signals. First, in order to have a triangulation to locate the seismo-atmospheric perturbation for the FM Sendai, we have installed one more station in Kanazawa, to be coordinated with our pre-existing station at Chofu. Other paths are (1) FM Shizuoka to Wakayama and (2) Osaka station to Kagoshima. This VHF system is reported, together with some preliminary results for a recent rather big earthquake (2004 Off Kii-peninsula earthquakes).
\end{abstract}

Key words: VHF signal, over-horizon reception, earthquakes, seismo-atmospheric perturbation

\section{Introduction}

There have been recently accumulated a lot of evidences on the presence of precursory electromagnetic phenomena of earthquakes (Hayakawa, 1999; Hayakawa and Molchanov, 2002). So, the electromagnetic phenomena might be of potential use in the short-term earthquake prediction (Hayakawa et al., 2004; Hayakawa, 2004). One of the three categories of seismo-electromagnetic observation is the use of transmitter signals to study the perturbations occurring in the atmosphere and/or ionosphere associated with earthquakes (Hayakawa, 2001). This method has a few advantages; the 1st is that any earthquakes near the propagation path from the transmitter to the observing point (receiver) may result in the propagation anomalies. In other words, this integrated observation enables us to accumulate a lot of data very easily. The second point is that the detailed information on the transmitter is well known beforehand, so that we can estimate the propagation anomaly in quantitative way. Any transmitter in different frequency ranges can be used. First, we know that subionospheric VLF/LF propagation is already known to be very effective in studying the lower ionospheric responses to the earthquakes (i.e., Hayakawa et al., 1996; Molchanov and Hayakawa, 1998; Molchanov et al., 2001). A lot of convincing results have been obtained not only in Japan, but also in different countries. Also, we have installed world-wide VLF network in Taiwan and in South Europe (Italy and Greece). The present paper deals with the higher frequency transmitter in VHF. Kushida and Kushida (2001) found some anomalous propagation in the VHF band which was associated with earthquakes. Then, Fukumoto et al. (2001) have performed the subsequent experiments by using the over-horizon reception at Chofu from the VHF transmitter in Sendai (FM Sendai), and have found that this anomalous propagation is likely to be associated with the anomaly in the atmosphere (not in the ionosphere) by means of the direction finding technique. Related works have been continued in this VHF band. Takano et al. (2004) have studied the broadband spectra in the VHF band, and have found a new phenomenon; reception of overseas (Thailand etc.) TV broadcasting waves possibly related to earthquakes. Yoshida et al. (2004) have been observing the radio emission in this VHF band.

In order to extend our VHF study, we have established a nation-wide VHF network in Japan. This paper reports on the detailed description of our VHF system, observing stations and we show some of our preliminary result for the recent Off Kii-peninsula earthquakes.

\section{Establishment of VHF network in Japan and system description}

Fig.1 illustrates a network for over-horizon VHF signal reception we have established in Japan. The transmitters are located in Sendai (FM Sendai), in Shimizu (FM Shizuoka) and in Osaka (Kansai 
International Media), and the corresponding receivers are located at Chofu, Kanazawa (for FM Sendai), at Wakayama (for FM Shizuoka) and at Kagoshima (for Kansai international Media). Table 1 indicates further information on both the transmitters (transmission frequency, transmitting power and propagation distance) and receiving stations.

\section{(a) Antenna and receiving systems}

The whole antenna system at each observing station is nearly the same as that used by Fukumoto et al. (2001). The polarization of the receiving antennas is only horizontal, and three 5-elements Yagi antennas are installed in the horizontal plane (parallel to the ground) with three different azimuthal direction $\left(-30^{\circ}\right.$ :west of the path, $0^{\circ}:$ along the path, $+30^{\circ}:$ east of the path). An additional Yagi antenna system is installed in the propagation direction (azimuth $=0^{\circ}$ ) with the elevation angle of $90^{\circ}$ (vertical). A combination of these antennas enables us to estimate the azimuthal and elevation angle of the incoming over-horizon signal (Fukumoto et al.; 2001). The signals detected by the above-mentioned Yagi antennas are fed to the FM tuners as shown in Fig. 2. The FM tuner used in this study is a commercial one: Panasonic Quartz Synthesizer FM/AM Tuner (ST-GT550), but we did the following important modification. That is, we have changed the ceramic filter for IF whose bandwidth is from $400 \mathrm{kHz}$ to $110 \mathrm{kHz}$ in order to enhance the separation from any neighbouring stations. And, we made it possible for us to monitor the wave electric intensity as an output of voltage.

The propagation paths for the three VHF transmitters are plotted in Figs. 3, 4 and 5. In Fig. 3 we intend to locate the atmospheric scatterer (or reflector) by means of the triangulation by using azimuths obtained at Kanazawa and Chofu. Fig. 4 refers to the path from Shizuoka to Wakayama, and Fig. 5 that from Osaka to Kagoshima.

(b) Data acquisition and transmission

Data transmission is of essential importance in this VHF network. An observing station is defined as the one responsible for data acquisition, while a master station is to collect the data observed at different observing stations. Fig: 6 shows the general schematic diagram for the master station (left) and for each observing (receiving) station (right). Fig. 7 indicates the system at each observing station. The data observed are handled by two PC's: the audio data are handled by one PC (recording) and the electric field data by different antennas are recorded on another PC. These two PC's have a function as a server, so that we can have an access to the data with a PC through the Internet. Let us add some more words on the network configuration. Data transmission is based on the home optical transmission line by NTT, and we do not use the fixed IP (Internet Protocol) address, but a random IP address. Though any server with service in the Internet has a definite IP address. We have solved this problem by using the DDNS (Dynamic Domain Name System) in such a way that using the domain name enables us to connect us to the PC of our interest even though the IP address of an observing station is changed. The DDNS client is included in the router.

Data transmission and remote operation of the observing system are performed with the use of SSH (Secure Shell), FTP (File Transfer Protocol), and a remote desk-top (Windows XP). The SSH is a remote shell with enhanced security, which enables us to control the whole operation of the sound of the transmitter signal. The FTP is used for the data transmission. By installing softwares in the FTP client at each observing station, we are able to get the necessary data at the master station. The remote desk-top is a service of Windows XP, which enables us to monitor, at the master station, a picture of the Windows PC at the observation station and to perform the operation at the master station.

For the observing station of Wakayama, we use the ADSL (Asymmetric Digital Subscriber Line) line by NTT. Again we do not use the fixed IP address, but use a random IP address given from the ISP (Internet Service Provider) side after the approval by PPPoE (Point to Point Protocol over Ether-net).

We need some special action to the observing station at Wakayama. Unlike other observing stations, this Wakayama station is completely un-manned, so we need some special treatment at this station. When the PC for the observation has some problems and it stops working, we can control the 


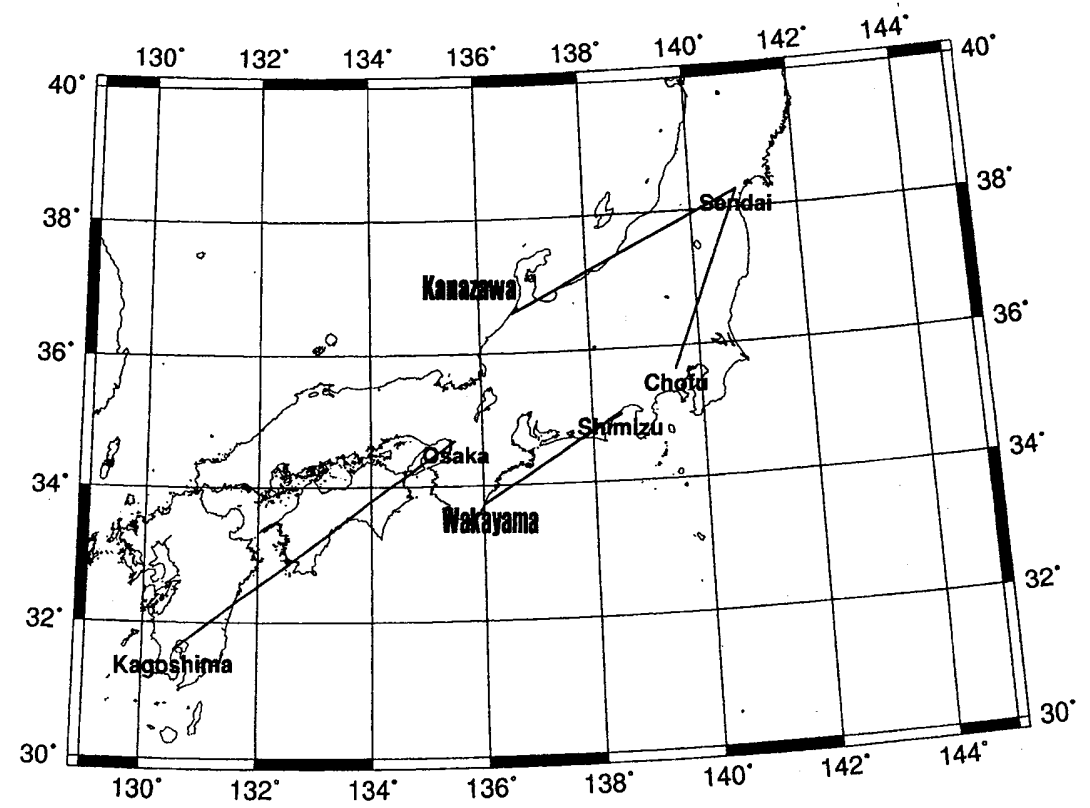

Fig. 1 A network of over-horizon VHF signals in Japan

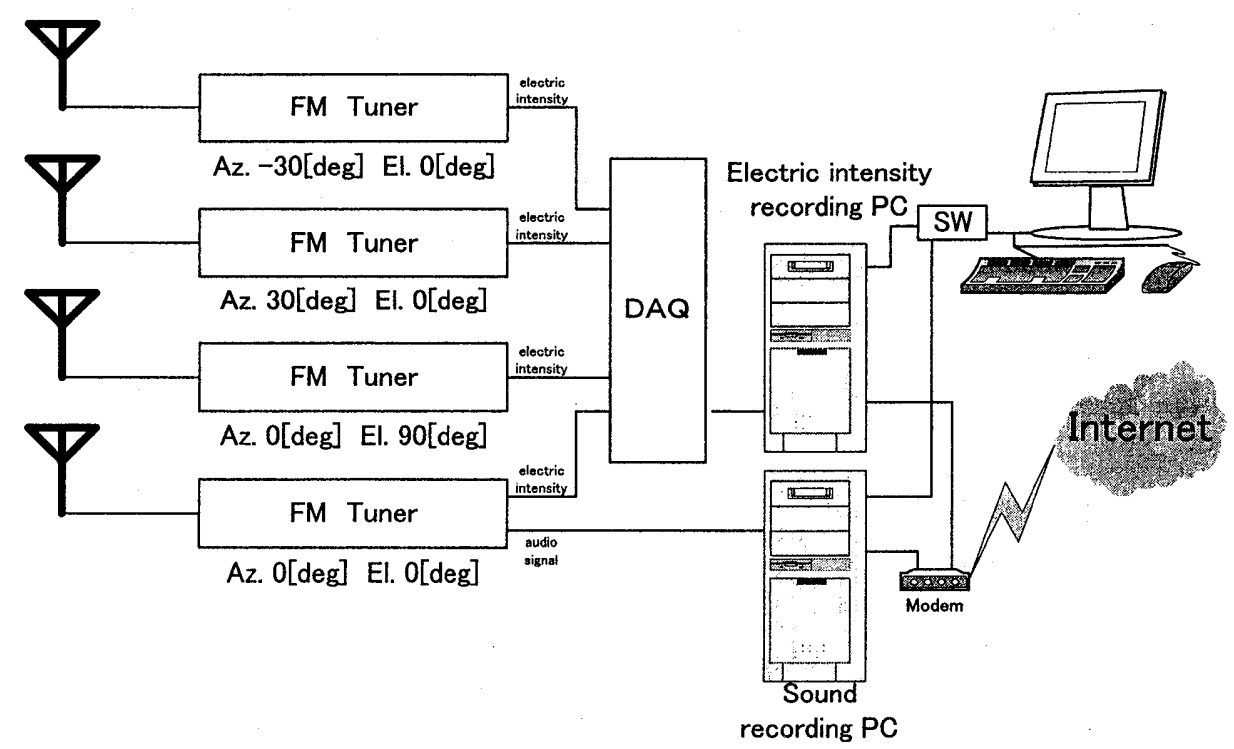

Fig. 2 Block diagram of VHF observing system at an observing station 


\begin{tabular}{|c|c|c|c|c|}
\hline $\begin{array}{c}\text { Observation } \\
\text { Station }\end{array}$ & $\begin{array}{c}\text { Transmitting } \\
\text { Station }\end{array}$ & Frequency & $\begin{array}{c}\text { Transmitting } \\
\text { Power }\end{array}$ & $\begin{array}{c}\text { Propagation } \\
\text { distance }\end{array}$ \\
\hline Chofu & $\begin{array}{l}\text { DateFM } \\
\text { Broadcasting } \\
\text { (FM Sendai) }\end{array}$ & \multirow[t]{2}{*}{$77.1 \mathrm{MHz}$} & \multirow[t]{2}{*}{$5 \mathrm{~kW}$} & \multirow[t]{2}{*}{$315 \mathrm{~km}$} \\
\hline $\begin{array}{l}\text { Lat. } 35^{\circ} 39^{\prime} 13^{\prime \prime} \\
\text { Long. } 139^{\circ} 32^{\prime} 39^{\prime \prime}\end{array}$ & $\begin{array}{l}\text { Lat. } 38^{\circ} 14^{\prime} 06^{\prime \prime} \\
\text { Long. } 140^{\circ} 52^{\prime} 32^{\prime \prime}\end{array}$ & & & \\
\hline Kanazawa & $\begin{array}{l}\text { DateFM } \\
\text { Broadcasting } \\
\text { (FM Sendai) }\end{array}$ & \multirow{2}{*}{$77.1 \mathrm{MHz}$} & \multirow[t]{2}{*}{$5 \mathrm{~kW}$} & \multirow[t]{2}{*}{$370 \mathrm{~km}$} \\
\hline $\begin{array}{l}\text { Lat. } 36^{\circ} 33^{\prime} 59^{\prime \prime} \\
\text { Long. } 136^{\circ} 39^{\prime} 51^{\prime \prime}\end{array}$ & $\begin{array}{l}\text { Lat. } 38^{\circ} 14^{\prime} 06^{\prime \prime} \\
\text { Long. } 140^{\circ} 52^{\prime} 32^{\prime \prime}\end{array}$ & & & \\
\hline Wakayama & $\begin{array}{l}\text { Shizuoka } \\
\text { FM } \\
\text { Broadcasting }\end{array}$ & \multirow[t]{2}{*}{$79.2 \mathrm{MHz}$} & \multirow[t]{2}{*}{$1 \mathrm{~kW}$} & \multirow[t]{2}{*}{$275 \mathrm{~km}$} \\
\hline $\begin{array}{l}\text { Lat. } 33^{\circ} 42^{\prime} 54^{\prime \prime} \\
\text { Long. } 136^{\circ} 00^{\prime} 10^{\prime \prime}\end{array}$ & $\begin{array}{l}\text { Lat. } 34^{\circ} 58^{\prime} 11^{\prime \prime} \\
\text { Long. } 138^{\circ} 28^{\prime} 22^{\prime \prime}\end{array}$ & & & \\
\hline Kagoshima & $\begin{array}{l}\text { Kansai } \\
\text { Intermedia } \\
\text { Broadcasting }\end{array}$ & \multirow[t]{2}{*}{$76.5 \mathrm{MHz}$} & \multirow[t]{2}{*}{$10 \mathrm{~kW}$} & \multirow[t]{2}{*}{$575 \mathrm{~km}$} \\
\hline $\begin{array}{l}\text { Lat. } 31^{\circ} 34^{\prime} 11^{\prime \prime} \\
\text { Long. } 130^{\circ} 33^{\prime} 51^{\prime \prime}\end{array}$ & $\begin{array}{l}\text { Lat. } 34^{\circ} 40^{\prime} 30^{\prime \prime} \\
\text { Long. } 135^{\circ} 40^{\prime} 54^{\prime \prime}\end{array}$ & & & \\
\hline
\end{tabular}

Table 1 Summary of VHF transmitters and receiving stations

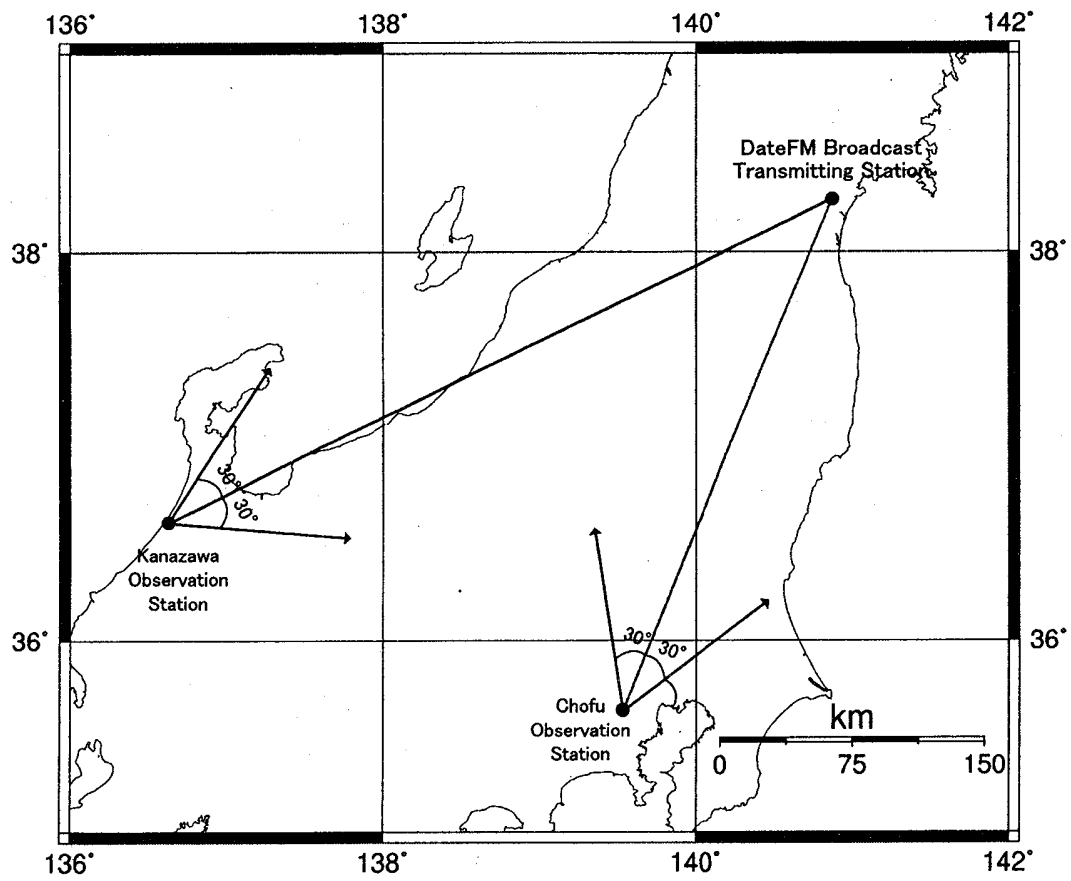

Fig. 3 A transmitter of FM Sendai and two observing stations (Kanazawa and Chofu) 


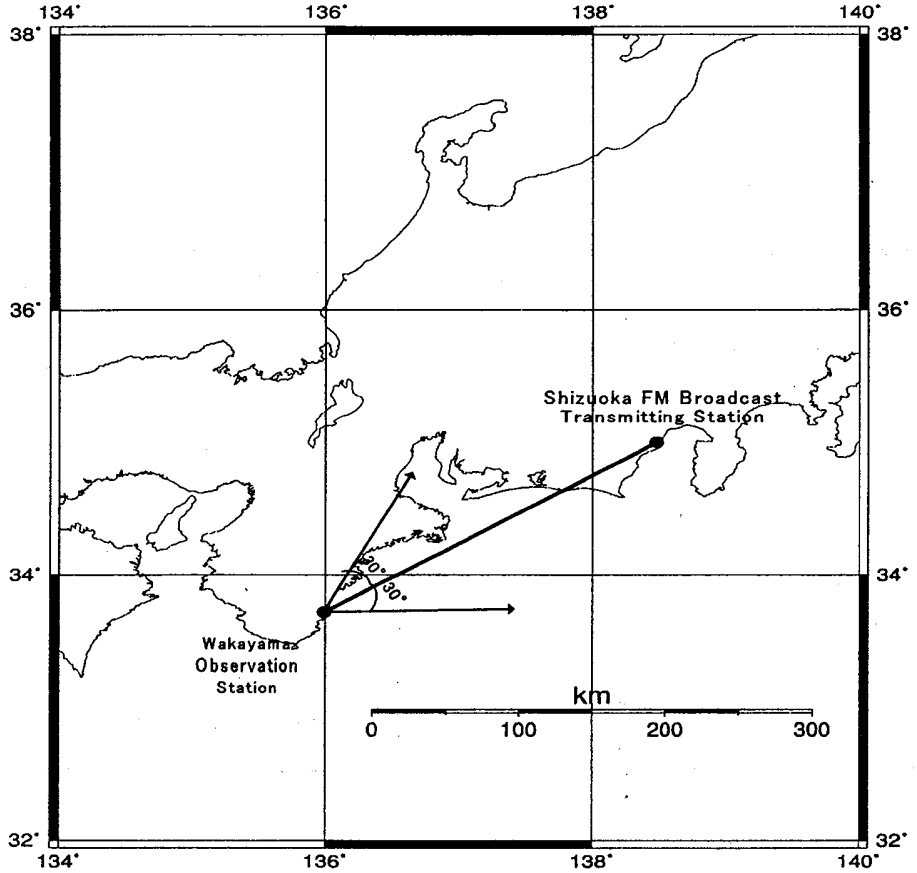

Fig. $4 \mathrm{~A}$ transmitter of Shizuoka FM and one receiving station of Wakayama

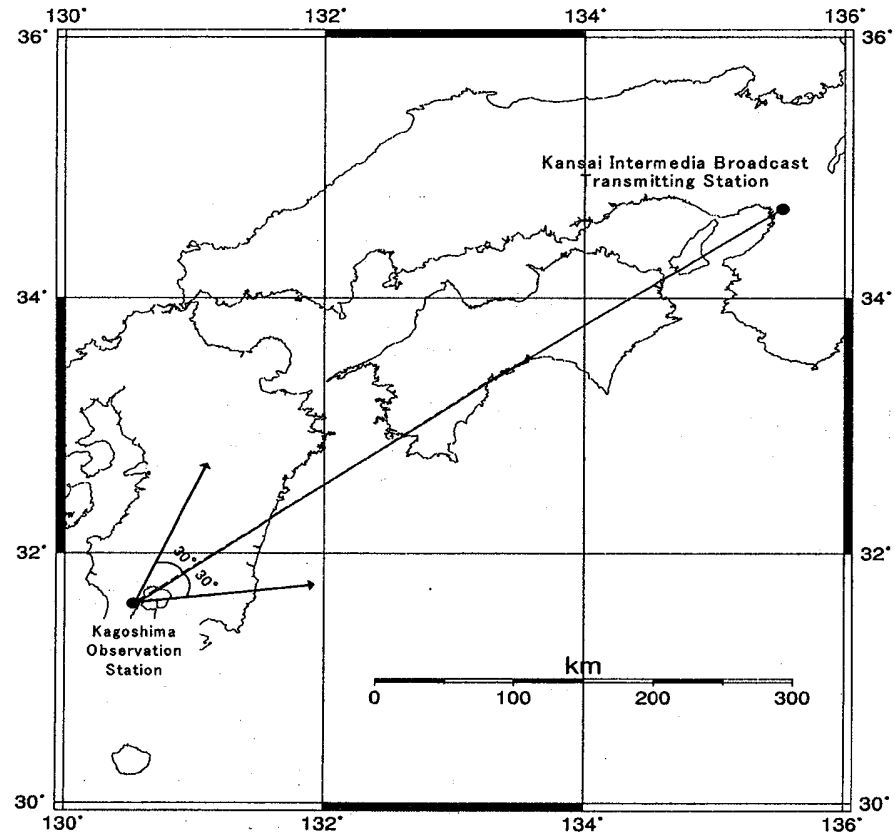

Fig. 5 A transmitter of Kansai Intermedia Broadcast (in Osaka) and an observing station at Kagoshima 


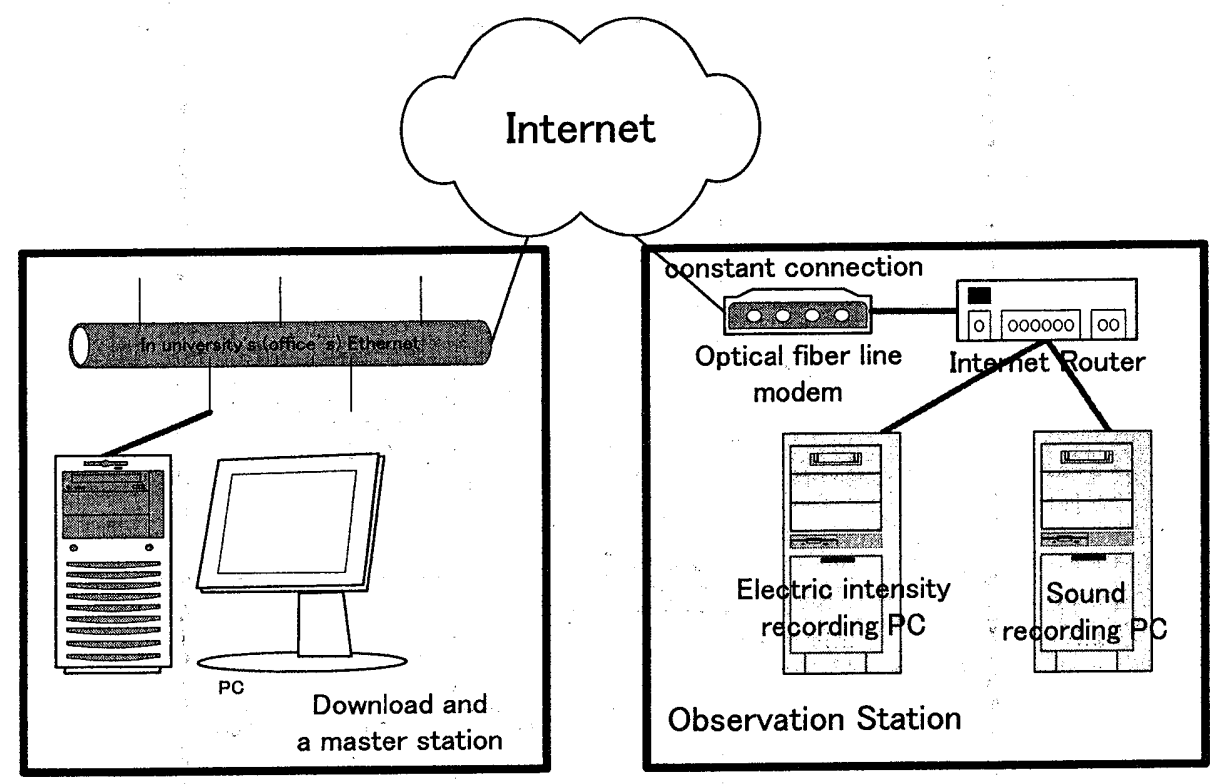

Fig. 6 Block diagram of network system

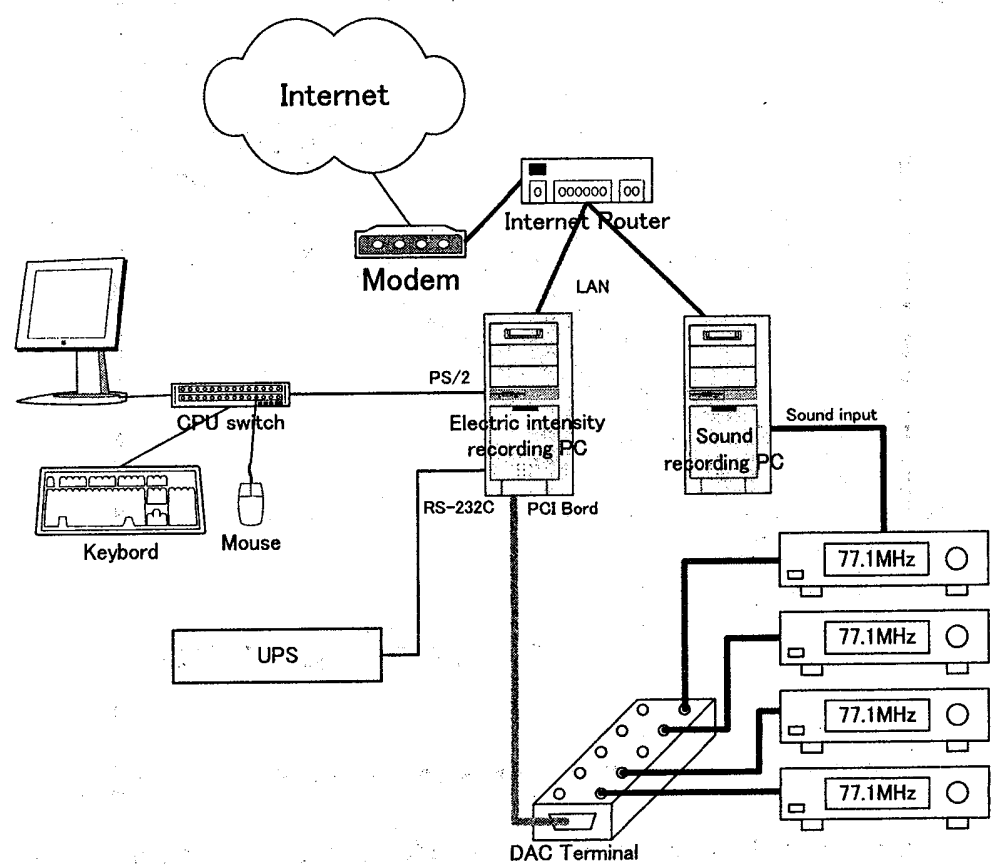

Fig. 7 Data acquisition and data transmission system (at each observing station) 

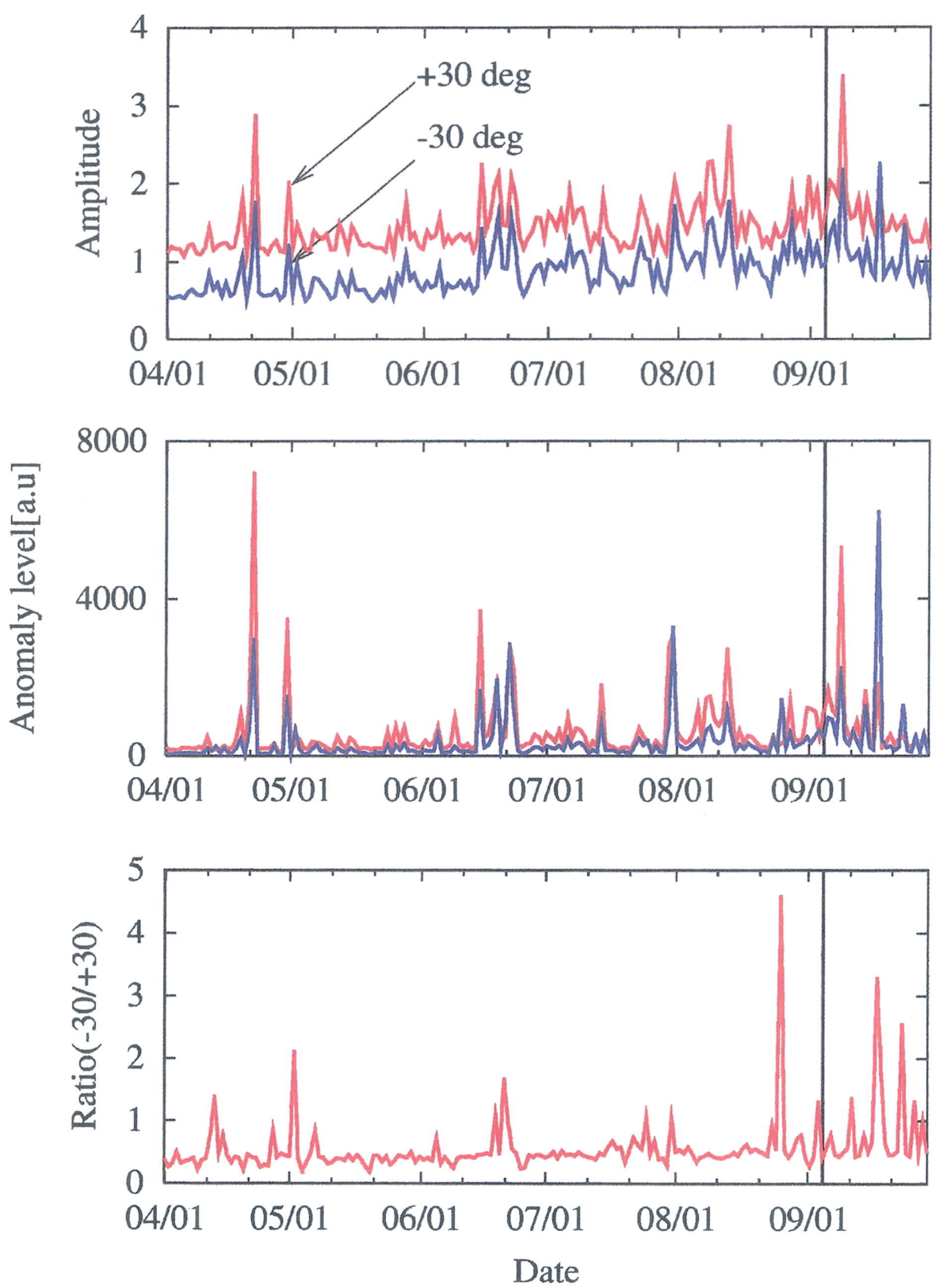

Fig. 8 An example of the VHF data (Shizuoka FM signal observed at Wakayama). The top panel illustrates the daily variation of the signal intensity for two azimuths $\left(+30^{\circ}\right.$, east of the path and $-30^{\circ}$, west of the path). The middle indicates the temporal of the anomaly level (in a. u.), in the east directions. The bottom is the temporal evolution of the ratio of the outputs for two azimuths $\left(-30^{\circ}\right.$ and $\left.+30^{\circ}\right)$. The vertical line (in black) is the time of the main shock on September 5. 


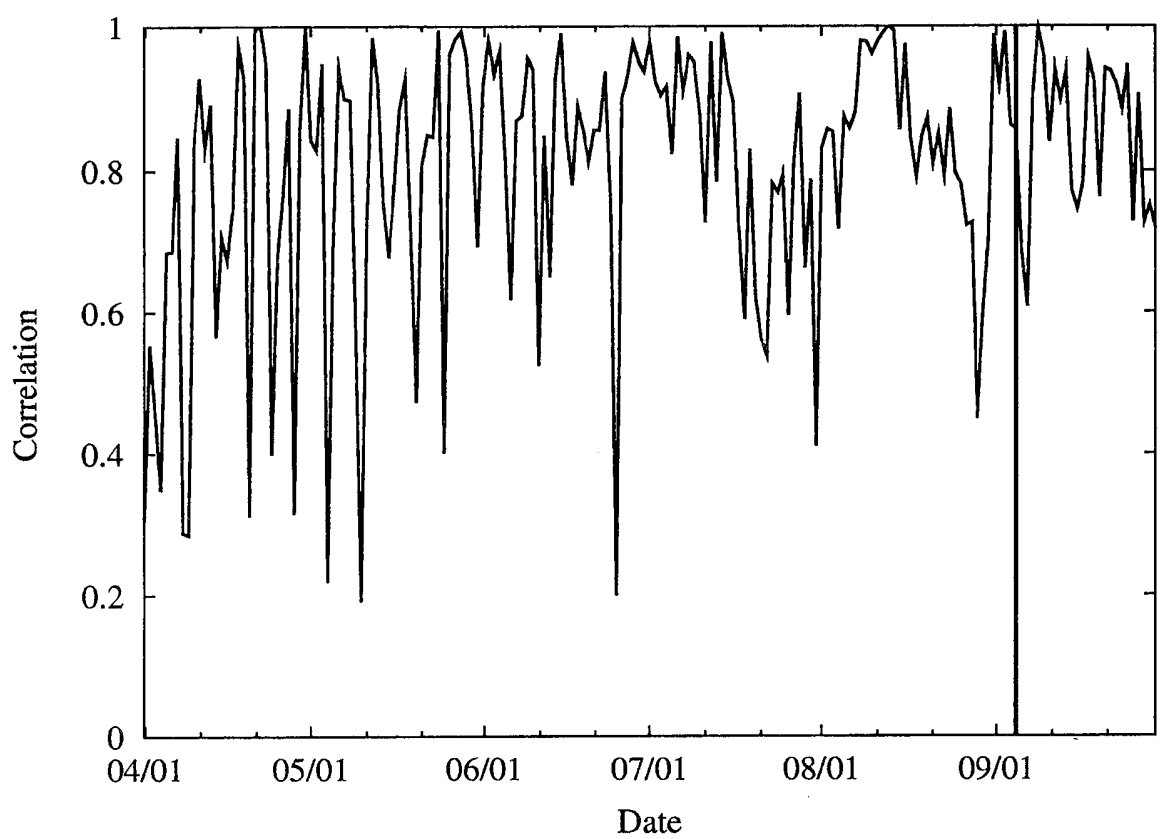

Fig. 9 Temporal evolution of the correlation between the outputs from the west and east directions of the path

operation of $\mathrm{PC}$ power and reset the operation through the telephone line.

\section{Some preliminary results : Off Kii-peninsula earthquakes}

We did the installation of this overall VHF system one by one. We started the observation at Kanazawa and at Wakayama in December 2003, but the observation was started at Kagoshima in August 2004. This means that the whole observations at Chofu, Kanazawa and Wakayama are going on since December 2003. During this short period, there occurred a rather large earthquake in the off-sea of Nagoya (named Off Kii-peninsula earthquak) on September 5, 2004. This earthquake had its magnitude of $\mathrm{M}=7.2$ and its depth was $100 \mathrm{~km}$. The foreshock occurred on September 5, 2004, 19: 07: 07 L.T. at the geographic coordinates $\left(33.05^{\circ} \mathrm{N}, 167.78^{\circ} \mathrm{E}\right.$ ) (with $\mathrm{M}=6.9, \mathrm{~d}=15 \mathrm{~km}$ ), and the main shock occurred at $\left(33.17^{\circ} \mathrm{N}, 137.14^{\circ} \mathrm{E}\right)$ on the same day at 23: 57: $17 \mathrm{~L}$.T. (with $\mathrm{M}=7.1, \mathrm{~d}=25 \mathrm{~km})$. An aftershock took place on September 7 (08: 29: 36 L.T.) at $\left(33.19^{\circ} \mathrm{N}\right.$, $\left.137.34^{\circ}\right)(\mathrm{M}=6.4, \mathrm{~d}=41 \mathrm{~km})$.

A comparison of the outputs from different antennas indicates that the output is much more enhanced for the elevation angle $0^{\circ}$ (horizontal) than for the elevation angle of $90^{\circ}$ (vertical upwords). This is likely to be consistent with our previous result by Fukumoto et al. (2001), and is indicative again of the presence of perturbation in the atmosphere, not in the ionosphere. Then, we pay attention to the outputs from a few antennas directed horizontally. Fig. 8 is the summary of the observation at Wakayama for the reception of over-horizon Shizuoka FM. The top panel illustrates the daily amplitude for the antenna directed west of the path $\left(-30^{\circ}\right)$ (in blue) and that directed east of the path $\left(+30^{\circ}\right)$ (in red). One value is assigned for one day, by taking the mean value of the electric field intensity during the current day. One more important point to mention here is that we have confirmed that this observed signal is just coming from 
the FM Shizuoka by means of the aural monitoring of its audio signal. The second panel is another presentation in which only the electric field intensity exceeding the mean plus $2 \sigma$ ( $\sigma:$ standard deviation) is taken, squared and integrated over the current one day. The bottom panel is the ratio of anomalous power for the azimuths of $-30^{\circ}$ and $+30^{\circ}$. This procedure enables us to deduce any anomalous behaviour of over-horizon VHF signal reception in much clearer way. The bottom panel of Fig. 8 indicates the presence of a clear anomaly on August 26, 2004, about 10 days before the earthquake. Fukumoto et al. (2001) found that the reception of anomalous over-horizon VHF signals is observed a few days to about one week before an earthquake. So, it may be possible that the peak in the bottom of Fig. 8 is a precursory signature of the earthquake. However, we need more detailed analyses before the definite conclusion on this peak..

Another quantity is used in Fig. 9, in which we have plotted the correlation coefficient between the temporal evolution of the electric field in the antenna $\left(-30^{\circ}\right)$ and that in the antenna $\left(+30^{\circ}\right) .120 \mathrm{sec}$ sampling data are used, though the initial data are observed every 10 seconds. You notice a clear indication of a periodicity of the order of one week during the whole observation period. However, it is easy for us to find some conspicuous disturbances in this periodical structure in August 2004. This might be some additional indication of the precursory effect.

\section{Acknowledgement}

The establishment of this VHF network is partly supported by Hōsō-Bunka Foundation, to which we are grateful. The plotting of Fig. 4 is done by Mr. S. Maekawa, to whom we are grateful for his help.

\section{References}

Fukumoto, Y., M. Hayakawa and H. Yasuda, Investigation of over-horizon VHF radio signals associated with earthquakes, Natural Hazards Earth System Sci., 1, $107 \cdot 112,2001$

Hayakawa, M. and Y. Fujinawa, Editors, Electromagnetic Phenomena Related to Earthquake Prediction, Terra Sci. Pub. Co., Tokyo, pp.667, 1994.

Hayakawa, M., O. A. Molchanov, T. Ondoh, and E. Kawai, The precursory signature effect of the Kobe earthquake on VLF subionospheric signals, J. Comm. Res. Lab., Tokyo, 43, 169-180, 1996.

Hayakawa, M., Editor, Atmospheric and Ionospheric Electromagnetic Phenomena Associated with Earthquakes, Terra Sci. Pub. Co., Tokyo, 996p, 1999.

Hayakawa, M., Electromagnetic phenomena associated with earthquakes: Review, Trans. Inst. Electr. Engrs. of Japan, 121-A, No.10, p.893-898, 2001.

Hayakawa, M. and O. A. Molchanov, Editors, Seismo Electromagnetics: Lithosphere Atmosphere - Ionosphere Coupling, TERRAPUB, Tokyo, 477p, 2002.

Hayakawa, M., Is earthquake prediction possible by means of electromagnetic phenomena? IEEJ (Inst. Electr. Engrs. Japan), Trans. Fundamentals and Materials, 124, No. 1, p. 3-4, 2004.

Hayakawa, M., K. Hattori and Y. Ando, Natural electromagnetic phenomena and electromagnetic theory: Review, IEEJ Trans. Fundamentals and Materials, 124, No. 1, p. 72-79, 2004.

Kushida, Y. and $R$. Kushida, Possibility of earthquake forecast by radio observations in the VHF band, Special Issue, "Seismo Electromagnetics", Ed. by M. Hayakawa, J. Atmos. Electr., 22, No.3, 239-255, 2002.

Molchanov, O. A., M. Hayakawa, T. Ondoh, and E. Kawai, Precursory effects in the subionospheric V.LF signals for the Kobe earthquake, Phys. Earth Planet. Inter., 105, 239-248, 1998. 
Molchanov, O. A., M. Hayakawa, and K. Miyaki, VLF/LF sounding of the lower ionosphere to study the role of atmospheric oscillations in the lithosphere-ionosphere coupling, Adv. Polar Upper Atmos. Res., 15,146-158, 2001.

Takano, T., Y. Yaji and K. Sakai, Spectrum analysis of anomalous propagation of FM radio broadcasting waves possibly related to earthquakes, Special Issue, "Seismo Electromagnetics", Ed. By M. Hayakawa, J. Atmos. Electr., 22, No.3, 257-265, 2002.

Yoshida, T., M. Nishi, T. Hatsuda, K. Kagosihma, and S. Obote, Observation of VHF radio emissions from the sun, Int. Symp. Antenna and Propagation, Aug. 17-21, Sendai, Japan, 2004.

(Received November 30, 2004; revised December 30, 2004; accepted January 13, 2005) 